\title{
La investigación en Enfermería: una mirada desde el enfoque de género'
}

Mtra. Edda R. Alatorre Wynter

${ }^{* *}$ Profesora de la ENEO-UNAM, Jefa de la División SUA.

A partir de algunos ejemplos, se evidencia la limitada participación de las mujeres en la actividad científica y se fundamenta cómo esta exclusión tiene su base en decisiones sociales androcéntricas y no en "limitaciones" biológicas. Se destaca la importancia del enfoque de género para hacer visible la discriminación a las mujeres en diversos ámbitos del hacer humano, entre ellos la ciencia. Finalmente, se traspolan estos argumentos al caso concreto de la investigación en la Enfermería y se hacen algunas propuestas viables de ser llevadas a cabo, entre otras, en la Escuela Nacional de Enfermería y Obstetricia de la UNAM.

PALABRAS CLAVE: Investigación y genero. Enfermería y género.

\section{Abstract}

Considering the corresponding history, androcentric decisions, not biological "limitations", have been the base for the exclusion of women from social activities such as science. Here, the perspective of gender is highlighted as one bridge to make this discrimination clearly visible. Also, the specific case of Nursing Research is considered by the National School of Nursing and Obstetrics (ENEO) of the National Autonomous University of Mexico (UNAM) along with some proposals for possible solutions

KEY WORDS: Research and gender. Nursing and gender.

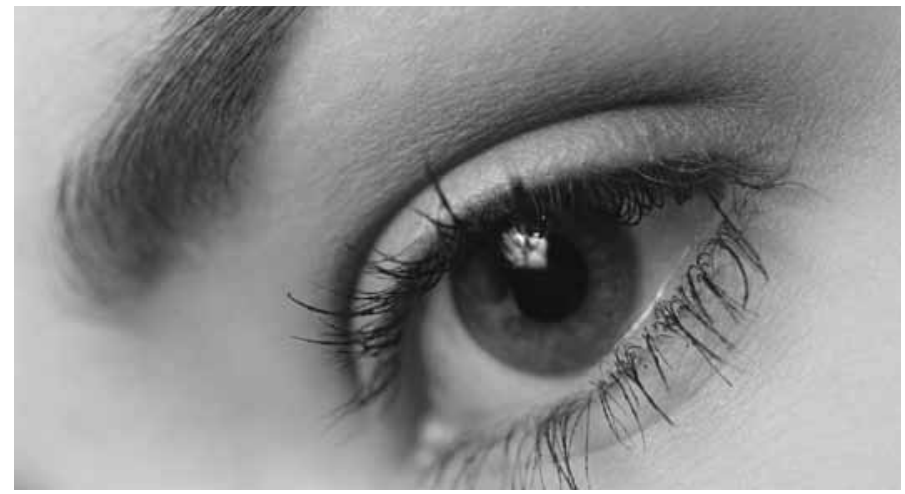

"Es fundamental que la Universidad Nacional establezca, como parte de una política institucional, el enfoque de género en todas sus acciones sustantivas. Debe hacerse una revisión crítica en el ámbito de la investigación, tanto en las ciencias exactas como en las ciencias sociales y en humanidades para incorporar los elementos que aporta el enfoque de género, lo que enriquece y amplía el conocimiento" 


\section{INTRODUCCIÓN}

En 1903, Marie Curie se convirtió en la primera mujer en ganar un Premio Nóbel (compartido con su esposo Pierre Curie y con Henri Becquerel) por su trabajo sobre la radioactividad. M. Curie recibió en 1911 un segundo Premio Nóbel, acontecimiento nunca antes visto, por descubrir el polonio y el radio.

Hace ya más de un siglo de esos acontecimientos; sin embargo, hoy, menos del 5\% de estos galardones son otorgados a mujeres. Es obvio que "el trabajo de las mujeres en el campo científico resulta poco recompensado", como señala Juan de Ávila (La Jornada $1^{\circ}$. de noviembre 1999) al hacer un recorrido al respecto, desde la creación del Premio Nóbel.

En la historia de cada una de las pocas mujeres científicas reconocidas hay una constante: diversas formas de discriminación y de bloqueo por atreverse a incursionar en un terreno limitado para ellas: la ciencia.* Cuántas otras historias habrá de mujeres estudiosas, observadoras, que sistematizaron sus hallazgos pero que ni siquiera pudieron ser vistas u oídas. Un ejemplo lo tenemos en Rosalind Franklin (1921-1958): "la heroína olvidada del ADN" quién desde que tenía 15 años de edad resolvió convertirse en científica, maravillada por lo que aprendía de la física y la química en una de las pocas escuelas inglesas, con menos discriminación, donde se permitía que a las niñas se les enseñaran estas materias. Entre 1951 y 1953, R. Franklin estuvo muy cerca de resolver por sí misma la estructura del ADN, pero murió de cáncer en 1958 a la edad de 37 años, cuatro años antes de que Watson, Crick y Wilkins recibieran el Premio Nóbel por sus aportaciones al estudio del ADN. ${ }^{1}$

\section{Mirando en torno de la discriminación.}

Sobre la poca o nula presencia de las mujeres en el ámbito de las ciencias se han dado las mas variadas explicaciones, centradas, la mayoría de ellas, en una "incapacidad natural" al conocimiento científico pues "la naturaleza las dotó" de mejores elementos para lo afectivo, lo doméstico, el cuidado y la protección, lo intuitivo; en cambio a los hombres, también "la naturaleza los dotó" de mejores elementos para el razonamiento, la acometividad, la creatividad y la toma de decisiones significativas.

Hay en estos planteamientos una clara adjudicación arbitraria de atributos, de comportamientos y de ámbitos de acción para los hombres y para las mujeres, sexualizados y jerarquizados desde un sistema androcéntrico que, to- mando al hombre como paradigma de lo humano, otorga más valor a los hombres y a lo masculino, en detrimento de las mujeres y lo femenino.

Esta última explicación se inscribe en otra forma de ver la realidad social; es decir, explicando que la distribución de tareas para cada sexo no se desprende directamente de las diferencias biológicas, sino que se construye como un hecho social determinado. La realidad social es entonces analizada desde el enfoque de género como herramienta para el análisis crítico del discurso androcéntrico. Esta forma de análisis, como apunta el Dr. Juan Ramón De la Fuente, enriquece y amplía el conocimiento (Gaceta UNAM mayo 2000). Nosotras agregamos, nos permite hacer visible la presencia y las contribuciones de las mujeres en la tarea humana de explicarnos el universo y las relaciones sociales en las que se construyen las ciencias y se toman las decisiones sobre su naturaleza e importancia.

\section{Participación igualitaria, isolución?}

Hace apenas dos años, en el V Congreso Iberoamericano de Ciencia celebrado en México en la Ciudad Universitaria se señaló que:

"La falta de equidad (que viven las mujeres) se manifiesta en la existencia de una especie de segregación territorial, traducida en la escasa presencia femenina en determinadas ramas y disciplinas del conocimiento y de la ciencia (en consecuencia) se aprecia un tipo de discriminación jerárquica para acceder a estos espacios" (Gaceta UNAM 19 de febrero de 2004).

Esta denuncia no es reciente. La historia de las luchas femeninas documenta diversos pronunciamientos por la equidad en todos los ámbitos del actuar humano. En el art. 5 inciso a) de la Convención para la Eliminación de Toda Forma de Discriminación a la Mujer (CEDAW) se establece que los Estados que han suscrito los preceptos de esta Convención -México entre ellos- se comprometen a:

"Modificar los patrones socioculturales de conducta de hombres y mujeres con miras a alcanzar la eliminación de los prejuicios y las prácticas consuetudinarias y de cualquier otra índole que estén basados en la idea de inferioridad y de superioridad de cualquiera de los sexos..."

En este sentido, no basta con establecer que las mujeres tengan las mismas condiciones que los hombres para acceder al conocimiento y al ejercicio científico pues puede resultar discriminatorio para las mujeres como bien advierte Alda Facio. 
"Si reconocemos que las mujeres y los hombres vivimos en condiciones distintas y desiguales (...) es obvio que tendremos necesidades diferentes; y por ende, una ley que parte de que somos iguales, que nos trata como si estuviéramos en igualdad de condiciones, no puede menos que tener efectos discriminatorios". ${ }^{2}$

La ciencia no surge del vacío social, ni es ajena a las relaciones de poder. Las mujeres, por lo ya expuesto, enfrentan condiciones asimétricas de poder en cualquier circunstancia. Se trata entonces, de construir e impulsar Políticas de Diferenciación para alcanzar la Equidad, es decir, igualdad en la diferencia. Las políticas y las diferentes acciones en materia investigativa "neutrales" dejan, en muchos aspectos, en desigualdad a las mujeres, porque en su formulación lo masculino sigue siendo el referente y porque suponen que se trata de que las mujeres "se igualen "a los hombres, que investiguen como los hombres. De lo que se trata es de eliminar lo masculino como paradigma de la igualdad. Mirar la investigación desde este enfoque abre la posibilidad de una forma alternativa de conocimiento, de cuestionar y trascender el monopolio masculino en la producción del conocimiento científico, de crear nuevos conocimientos sobre viejos temas, y de formular renovados argumentos y recursos interpretativos. ${ }^{3}$

Al respecto, Roberto Castro y Mario Bronfman concluyen que:

\footnotetext{
"cuando se habla de la posibilidad de una nueva ciencia feminista, no se hace referencia a un tipo de conocimiento que sólo las mujeres, por ser mujeres, pueden reproducir [...] lo que se está proponiendo es que una nueva estructura social, sin dominación de género, dará lugar a formas de conocimiento que antes permanecían suprimidas o reprimidas [...] Es en este tipo de conocimiento insospechado donde radica una de las mayores promesas de la teoría feminista". ${ }^{4}$
}

En efecto, se trata de aceptar un hecho inobjetable, el mundo está habitado por mujeres y por hombres y ambos tienen mucho que aportar para conocerlo; hasta hoy, la única visión que ha prevalecido es la masculina, aunque provenga aun de las mismas mujeres, instruidas también desde una visión masculina.

\section{¿Qué aporta el enfoque de género a la investiga- ción que hacen las Enfermeras?}

Hoy, decir Enfermería es decir mujeres. Aunque la profesión puede ser estudiada también por hombres, lo cierto es que en todo el mundo ellos son todavía una minoría.
En México, aunque varían los porcentajes por institución educativa y por entidad, el promedio de mujeres Enfermeras constituye más del 90\%.

Hasta este momento hemos reflexionado sobre diversos mecanismos sociales que explican la marginación y aún la exclusión de las mujeres en el quehacer científico.

Entonces, si las Enfermeras son en su gran mayoría mujeres es obvio apuntar que su presencia en la ciencia tampoco es visible.

Analizar la Enfermería desde este enfoque, es tener los elementos para fundamentar una manera feminista alternativa, no excluyente de pensar, de observar, de explicar y de actuar en el área de la salud. Producto a su vez, de una educación y un desarrollo investigativo donde no prevalezca el enfoque e interés médico hegemónico.

Investigar con perspectiva de género permite trazar las coordenadas de nuevos campos de estudio; demostrar por ejemplo, que cuando se incluye en las variables sólo el sexo: masculino o femenino, se está señalando solamente una pertenencia biológica, no una valoración social; esta última, el género, no puede estar ausente pues seguiríamos explicando cualquier fenómeno desde la perspectiva masculina.

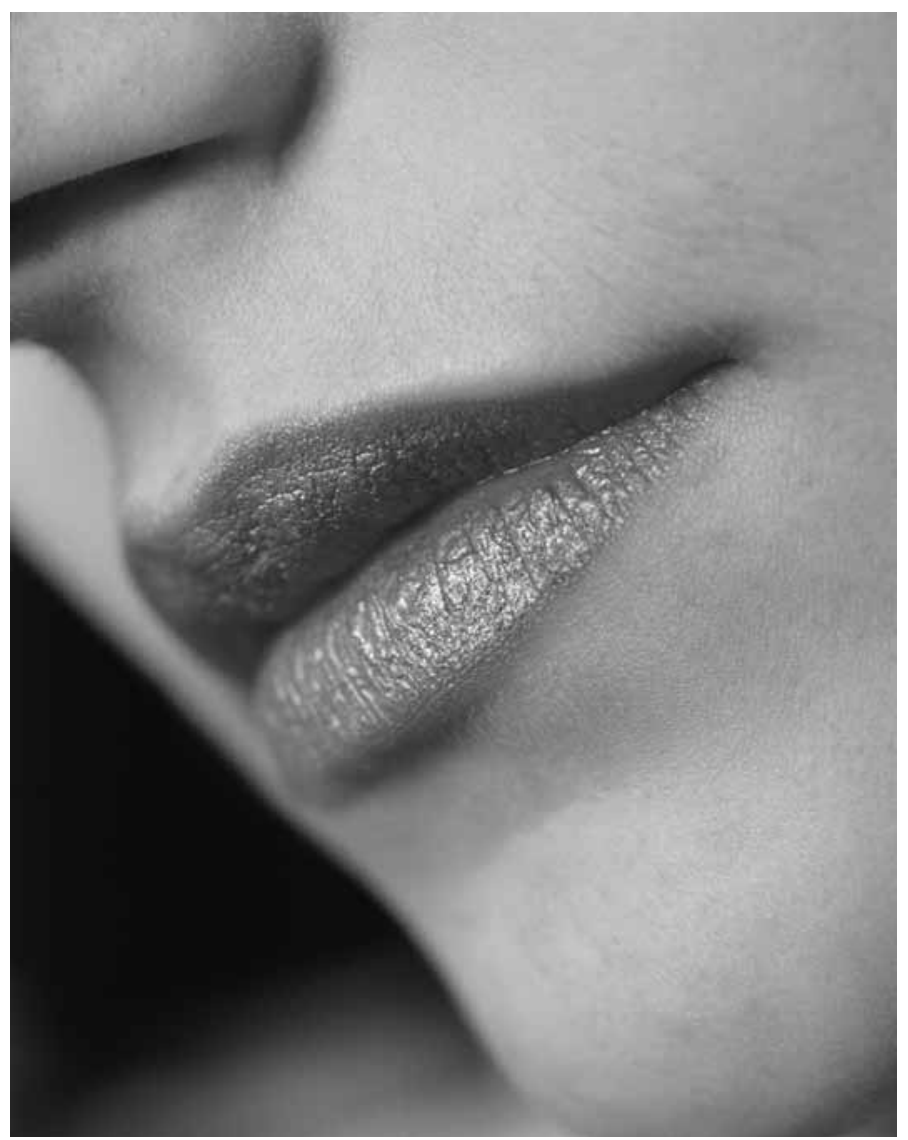


Cuando se investiga un fenómeno social en el área de la salud, con enfoque de género, se está explicando cómo interactúa cualquier categoría: edad, trabajo, escolaridad, familia, preferencia sexual, raza, estado civil, etc. con las construcciones de género, para definir los conocimientos y las prácticas de la salud y de la enfermedad, y para conocer el grado de autonomía o de dependencia que pueden tener las mujeres y los hombres sobre su propio cuerpo, su sexualidad, su libertad, sus posibilidades para acceder a los recursos económicos, al conocimiento y al poder.

Decir niño no es decir niña, decir anciano no es decir anciana, decir enfermo no es decir enferma, decir deprimido no es decir deprimida, decir investigador no es decir investigadora, decir hombre no es decir mujer. El pertenecer al género femenino constituye un mayor riesgo para la salud de las mujeres, y constituye también un mayor obstáculo para el desarrollo científico de las mujeres. Premisas fundamentales para las Enfermeras investigadoras.

\section{Propuestas.}

En consecuencia con lo planteado hacemos dos grandes propuestas. Cada una de ellas requerirá a su vez ser desarrollada con más amplitud en el seno de las academias.

1.- Construir e impulsar políticas de diferenciación en la UNAM, por ejemplo:

- No restringir, por edad, a las mujeres que soliciten beca para estudiar posgrados. Los tiempos de desarrollo académico suelen ser más lentos en ellas por razones de maternidad y de crianza, lo que las limita también en la actividad investigativa.

- Impulsar y diversificar la formación de posgrado y con esto la formación investigativa a través de diversas modalidades en SUA.

- Ofrecer formación teórica y metodológica en estudios de género para docentes y alumnas(os).

- Promover y estimular las investigaciones que aborden temas sobre equidad de género y Derechos Humanos.

2.- Apoyar y fortalecer la línea de investigación de la ENEO sobre Estudios de Género.
- Promover en los diferentes seminarios optativos de la ENEO la perspectiva de género.

- Promover la perspectiva de género como una herramienta para el análisis crítico y la argumentación en las diversas investigaciones, sobre todo de corte cualitativo.

Estas propuestas y muchas más, sólo serán posibles cuando la ENEO cuente con presupuesto especial para actividades investigativas, de otra manera seguirán quedando sólo como buenas intenciones.

\section{REFERENCIAS BIBLIOGRÁFICAS.}

1. Durán, María Ángeles. "Liberación y utopía. La mujer ante la ciencia". En: Belausteguigoitia, Marisa. Mingo, Araceli. Géneros prófugos. Feminismo y Educación. UNAM.PUEG. CESU. colegio de las Vizcaínas. Piadós Género y Sociedad. México, 1999. p. 323-347.

2. Facio, Alda. Cuando el género suena cambios trae. Metodología para el análisis de género del fenómeno legal. ILANUD. San José, Costa Rica. 1992. p. 81

3. Cazés, Daniel. La perspectiva de género. CONAPO. México, 2000. p.37

4. Castro, Roberto y Mario Bronfman, . "Teoría Feminista y Sociología Médica: Bases para una discusión". En: Figueroa Perea, Juan G. (comp.). La condición de la mujer en el espacio de la salud. El Colegio de México. México 1998 p. 205-233.

\section{NOTAS}

* $\quad$ Este tema es fundamental para entender la condición social de las Enfermeras, pero escapa a los propósitos de este trabajo. Se sugiere revisar: Alatorre Wynter, Edda. El desarrollo histórico de la Enfermería en México. ENEO-UNAM 1985 y Alatorre Wynter, Edda. Hacia un nuevo perfil académico profesional en la Escuela Nacional de Enfermería y Obstetricia de la UNAM desde la perspectiva de género. FF y L. UNAM 2003.

\section{DIRECCIÓN PARA CORRESPONDENCIA:}

Edda Alatorre Wynter: alatorre7773@hotmail.com 